\title{
Towards next generation time-domain diffuse optics devices
}

\author{
Alberto Dalla Mora*a , Davide Contini ${ }^{\mathrm{a}}$, Simon R. Arridge ${ }^{\mathrm{b}}$, Fabrizio Martelli ${ }^{\mathrm{c}}$, \\ Alberto Tosi ${ }^{\mathrm{d}}$, Gianluca Boso ${ }^{\mathrm{d}}$, Andrea Farina ${ }^{\mathrm{e}}$, Turgut Durduran ${ }^{\mathrm{f}}$, \\ Edoardo Martinenghi ${ }^{\mathrm{a}}$, Alessandro Torricelli ${ }^{\mathrm{a}}$, Antonio Pifferi ${ }^{\mathrm{a}, \mathrm{e}}$ \\ ${ }^{a}$ Dipartimento di Fisica, Politecnico di Milano, Milano, Italy; \\ ${ }^{b}$ Department of Computer Science, University College London, London, United Kingdom; \\ ${ }^{\mathrm{c}}$ Dipartimento di Fisica e Astronomia, Università degli Studi di Firenze, Firenze, Italy; \\ ${ }^{\mathrm{d}}$ Dipartimento di Elettronica, Informazione e Bioingegneria, Politecnico di Milano, Milano, Italy; \\ ${ }^{\mathrm{e}}$ Istituto di Fotonica e Nanotecnologie, Consiglio Nazionale delle Ricerche, Milano, Italy; \\ ${ }^{\mathrm{f}}$ ICFO-Institut de Cències Fotòniques, Castelldefels (Barcelona), Spain.
}

\begin{abstract}
Diffuse Optics is growing in terms of applications ranging from e.g. oximetry, to mammography, molecular imaging, quality assessment of food and pharmaceuticals, wood optics, physics of random media. Time-domain (TD) approaches, although appealing in terms of quantitation and depth sensibility, are presently limited to large fiber-based systems, with limited number of source-detector pairs. We present a miniaturized TD source-detector probe embedding integrated laser sources and single-photon detectors. Some electronics are still external (e.g. power supply, pulse generators, timing electronics), yet full integration on-board using already proven technologies is feasible. The novel devices were successfully validated on heterogeneous phantoms showing performances comparable to large state-of-the-art TD rackbased systems. With an investigation based on simulations we provide numerical evidence that the possibility to stack many TD compact source-detector pairs in a dense, null source-detector distance arrangement could yield on the brain cortex about 1 decade higher contrast as compared to a continuous wave $(\mathrm{CW})$ approach. Further, a 3 -fold increase in the maximum depth (down to $6 \mathrm{~cm}$ ) is estimated, opening accessibility to new organs such as the lung or the heart. Finally, these new technologies show the way towards compact and wearable TD probes with orders of magnitude reduction in size and cost, for a widespread use of TD devices in real life.
\end{abstract}

Keywords: time-domain diffuse optics, single-photon detectors, fast time gating, time resolved reflectance spectroscopy, diffuse optical imaging.

\section{INTRODUCTION}

Diffuse optics found interesting applications like imaging of brain function [1]-[3], oximetry [4], optical mammography [5], food [6] or wood [7] quality assessment, and characterization of pharmaceutical solids [8]. The most common technique is represented by Continuous-Wave (CW) approach [3]. In this case the equipment is typically compact and cheap, but shows poor performances [9],[10] since: $i$ ) with a single measurement it is not possible to uncouple absorption from scattering effect; ii) measurements cannot provide absolute values of the optical properties; iii) the mean penetration depth of detected photons increases by increasing the source-detector separation $\rho$, thus requiring to use large separations $(>2 \mathrm{~cm})$ to probe scattering media in depth. Vice versa, Time-Domain (TD) instrumentations do not suffer from the same limitations [11]-[13], thus also allowing the use of short $\rho$ [14]-[19] (or even null $\rho$ [20]) and thus permitting the most complete coverage of the sample by placing a large number of source-detector pairs. However, at small $\rho$, due to the huge increase in the number of detected weakly diffused photons, a fast time-gated detector is absolutely required to avoid the saturation of the detection chain [21]-[23]. Unfortunately, present technology TD systems are bulky and expensive, thus strongly limiting the number of measurement channels [1],[24].

In this work we will first introduce the potentialities of TD technique to show the ultimate physical limit for depth sensitivity. We will then present a first building block with the potential to enable to the design of next-generation diffuse optical devices.

Optical Tomography and Spectroscopy of Tissue XI, edited by Bruce J. Tromberg, Arjun G. Yodh,

Eva Marie Sevick-Muraca, Robert R. Alfano, Proc. of SPIE Vol. 9319, 93191J

(C) 2015 SPIE · CCC code: $1605-7422 / 15 / \$ 18 \cdot$ doi: $10.1117 / 12.2078955$ 


\section{CONTRAST SIMULATIONS}

We first addressed the problem of the detection of an absorption inhomogeneity at a depth $z$ within a homogeneous medium. Results have been generated with a forward solver accounting for the effects of an absorption perturbation placed inside a diffusive slab based on the Born approximation [25] applied to the photon diffusion equation that has been solved with the extrapolated boundary condition [26]. We considered ideal sources and detectors with $2 \pi$ collecting solid angle. This is not a limitation of the results obtained with these simulations since within the diffusion approximation it is possible to show that the shape of the temporal and spatial distribution of exiting photons is independent from the angular receiving range of the detector [27]-[29]. All the results presented pertain to a diffusive regime of photon migration. In order to account for the actual area of sources and detectors we have implemented an integral of the absorption perturbation over the source surface (illumination distributed over an area of $1 \mathrm{~cm}^{2}$ ) and detector (active detecting area of $1 \mathrm{~cm}^{2}$ ) using an integration step of $2 \mathrm{~mm}^{2}$. A power density of $4 \mathrm{~mW} / \mathrm{mm}^{2}$ has been assumed. The acquisition time was set to $1 \mathrm{~s}$. A further integration was also performed over the volume of the absorbing inclusion that was divided into eight voxels.

For the calculations of the TD case we have assumed an absorbing inclusion of $1 \mathrm{~cm}^{3}$ set at several depths $z$ (i.e. 10, 20, $30,40,50,60 \mathrm{~mm})$ and the data have been plotted versus the detection time $t$. The inclusion having an absorption variation $\Delta \mu_{a}$ of $0.01 \mathrm{~mm}^{-1}$ with respect to the background was placed inside a semi-infinite medium with reduced scattering coefficient $\mu_{s}^{\prime}=1 \mathrm{~mm}^{-1}$, and an absorption coefficient $\mu_{a}=0.01 \mathrm{~mm}^{-1}$, with source and detector set right above the inclusion at $\rho=0$. We assumed to be able to detect the inclusion when a Poisson noise $\sigma<1 \%$ is present on the number of detected photons for the homogeneous case. Then, we calculated the maximum time $t_{\max }$ for a gate of $1 \mathrm{~ns}$ that permits to have 10 kcounts (Poisson noise $=1 \%$ ), thus obtaining $t_{\max }=9.6 \mathrm{~ns}$.

Since TD measurements are simulated at $\rho=0$, sources and detectors are totally overlapped in the $1 \mathrm{~cm}^{2}$ area. This, in principle, is not unfeasible. It is possible to envisage a two-layer device where the detectors occupy the lower layer and the sources the upper one. A set of small emitters (as for instance Vertical-Cavity Surface-Emitting Laser - VCSELs -) can fire through a hole or a transparent window in the detector plane. If the whole probe is separated from the medium by a few millimeters thick transparent foil, then the source can expand to provide a uniform illumination onto the tissue.

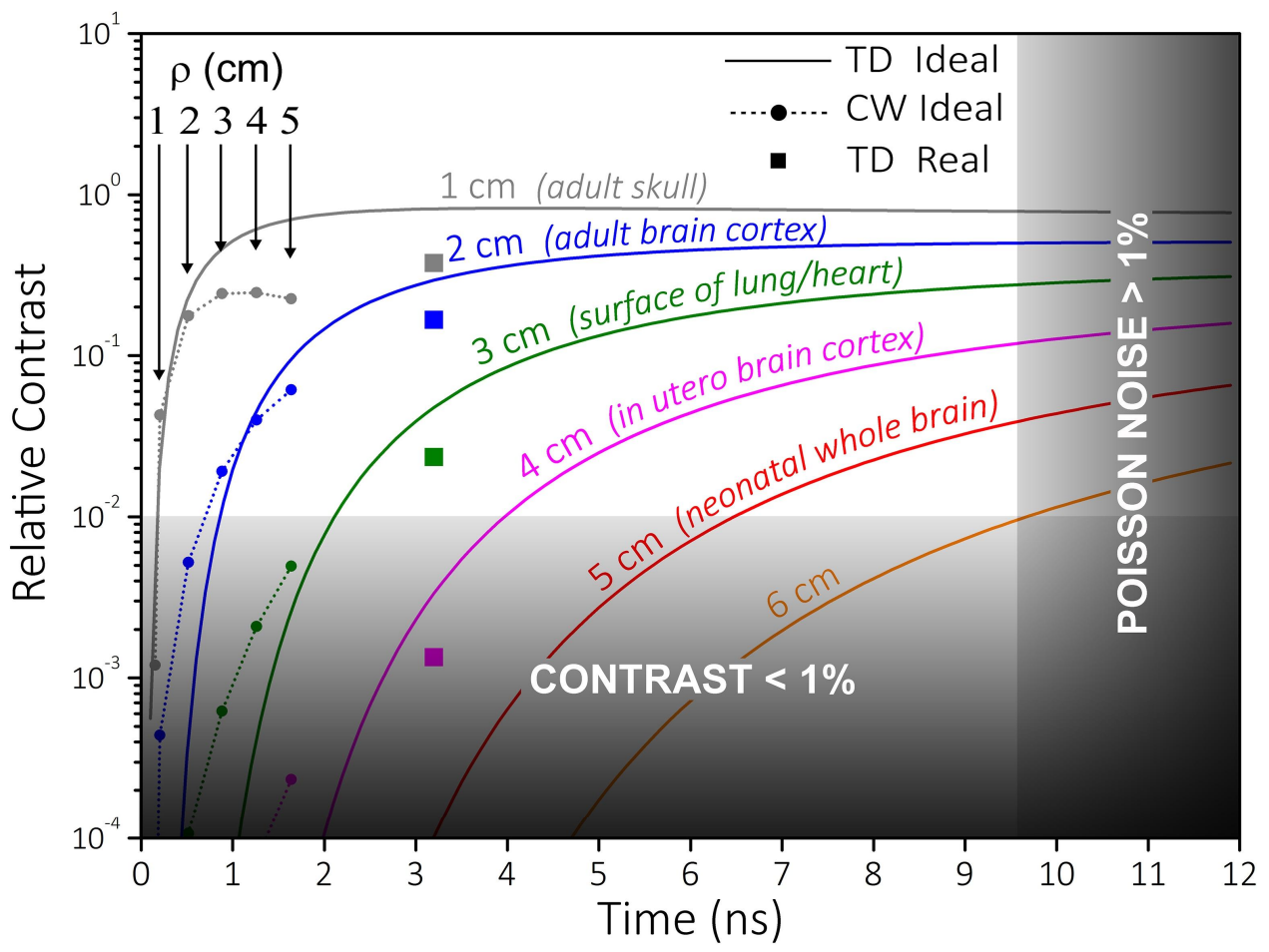

Figure 1. Simulations of the relative contrast produced by a $1 \mathrm{~cm}^{3}$ spherical perturbation set at different depths $z$ in a homogeneous medium with $\mu_{a}=0.1 \mathrm{~cm}^{-1}$ and $\mu_{s}^{\prime}=10 \mathrm{~cm}^{-1}$ related to: $i$ ) an ideal TD system; $\left.i i\right)$ an ideal CW system; and iii) a real (state-of-the-art) TD system [24]. 
Thus, just a small fraction of the detector plane is spoiled for letting the source out, and the $100 \%$ coverage can still be assumed as an ideal ultimate case. Moreover, for small values of $\rho$ (for instance $2 \mathrm{~mm}$ ) the time-resolved reflectance distribution, except for the very early photons, slightly differs from that obtained for $\rho=0$ [14]. For this reason, in TD the relative contrast achievable for $\rho=0$ is also achievable for small values of $\rho$ and this guarantees the feasibility of the simulated measurements, where an integrated area of $1 \mathrm{~cm}^{2}$ for the detector was assumed.

The calculations for the CW case have been done for the same optical and geometrical properties as for the TD calculations except for the source detector distance $\rho$ that was set at 10, 20, 30, 40 and $50 \mathrm{~mm}$.

The simulations have been obtained for a semi-infinite homogeneous medium. We have selected this geometry since we wanted to compare $\mathrm{CW}$ and TD in a simple geometry, to better understand the basic properties of the two approaches.

Figure 1 shows the relative contrast $C=\left(N_{0}-N\right) / N_{0}$ produced, where $N$ and $N_{0}$ are, respectively, the perturbed and unperturbed detected photons. In Figure 1 ideal CW and TD systems for different $z$ positions of the inclusion are compared. As explained before, we assumed $\rho=0$ for TD and a variable $\rho$ for $\mathrm{CW}$. In this latter case, $C$ is plotted against the average propagation time, which is dependent on the considered $\rho$. By assuming as criteria for detectability a $C>1 \%$ and a $\sigma<1 \%$ (i.e. the white region in Figure 1), a CW ideal system can detect the object only down to $2 \mathrm{~cm}$ when using $\rho>4 \mathrm{~cm}$ because of low contrast. Conversely, a TD ideal system can reach up to $6 \mathrm{~cm}$ around $t=10 \mathrm{~ns}$. In this case, the contrast in TD is $10^{4}$ times higher than with a CW ideal system. For comparison, a state-of-the-art TD system [24] operating at $\rho=3 \mathrm{~cm}$ (squares in Figure 1) can reach only $t=3 \mathrm{~ns}$, yielding a maximum $z=3 \mathrm{~cm}$.

We expect the key advantages of TD to show up also in heterogeneous geometries like a two-layer medium, being ultimately linked to the chance to increase the photon path-length in TD by extracting the few long-lived photons. Further, TD approaches could provide additional value when dealing with both absorption and scattering inhomogeneities, due to the intrinsic aptness of TD to disentangle the two contributions. Yet, these aspects were not investigated in the present work.

\section{TOMOGRAPHY SIMULATIONS}

TD diffuse optical tomography was simulated considering a cubic part of a semi-infinite homogeneous scattering medium with $60 \mathrm{~mm}$ side, $\mu_{a}=0.1 \mathrm{~cm}^{-1}, \mu_{s}^{\prime}=10 \mathrm{~cm}^{-1}$, and $\mathrm{n}=1.33$ refractive index. We considered sources and detectors with $10 \times 10 \mathrm{~mm}^{2}$ area, placed on the surface. A cubic perturbation (absorption coefficient twice the background, $10 \mathrm{~mm}$ side) was set at a depth of $40 \mathrm{~mm}$. TD and $\mathrm{CW}$ data were generated using the block diagram depicted in Figure 2. Homogeneous data was produced using Eqs. (4.16), (4.22) and (4.45) of [29]. Fluence rate data $\left(\Phi_{0}\right)$ were then converted to photon numbers $\left(N_{0}\right)$ assuming, similarly to the previous simulations presented, an exposure of $4 \mathrm{~mW} / \mathrm{mm}^{2}$, monochromatic radiation ( $800 \mathrm{~nm}$ wavelength) and an acquisition time of $1 \mathrm{~s}$. Detectors were again considered with a unitary quantum efficiency, acceptance solid angle of $2 \pi$ and a delta-like temporal response. Using Eqs. (7.25), (7.28) and (4.45) of [29], the Jacobian matrix $J$ was calculated and perturbed data $\left(\Phi_{p}\right.$ and $\left.N_{p}\right)$ were consequently generated. Then a transmission filter $\left(T_{\alpha}\right)$ was applied with the aim to simulate TD gated and CW measurements. Concerning TD gated measurements, the maximum value for single-photon counting was set at $1 \mathrm{MHz} / \mathrm{mm}^{2}$ assuming every detector composed by 10 by 10 single pixels $\left(1 \mathrm{~mm}^{2}\right.$ each) having its own count rate limit. This limit is set according to the limits shown in Figure 1. In a gated measurement the acquisition count rate during each temporal window is adjusted to the maximum level by changing the source power [30],[31], hence the following transmission filter is applied at each time: $T_{T D}=N_{M A X} / N_{0}$ when $N_{0} \geq N_{M A X}$ and $T_{T D}=1$ when $N_{0}<N_{M A X}$ (where $N_{M A X}$ is the maximum count rate. To make the comparison with the $\mathrm{CW}$ case feasible in total measurement time, the maximum limit of $1 \mathrm{MHz} / \mathrm{mm}^{2}$ has been evenly distributed for each gate. Thus, the limits were divided by the number of temporal windows used (about 100 windows of $100 \mathrm{ps}$ ). Since $\mathrm{CW}$ data are not limited by the single photon statistic, the filter $T_{C W}$ applied is unitary. These data were then perturbed by additive Gaussian noise $(\sigma=0.1 \%)$ to represent fluctuations of laser sources, detectors, electronics and other. Poisson noise was then further added to take into account the light fluctuations. After that relative contrasts ( $y$ in Figure 2) were calculated for each source-detector couple.

Once forward data were generated, reconstruction were performed by using the generalized minimal residual method [32] applied with a maximum of 150 iterations $\left(10^{-6}\right.$ tolerance) to the regularized normal equation $\left(J^{T} J+\lambda^{2} I\right) \Delta \mu_{a}=J^{T} J$ (where $J$ is the Jacobian matrix, $\lambda$ - set to 1 - is the parameter of regularization, $I$ is the identity matrix, and $\Delta \mu_{a}$ is the absorption variation in each voxel [33]. The volume was divided into $2.5 \mathrm{~mm}$ side cubic voxels. 


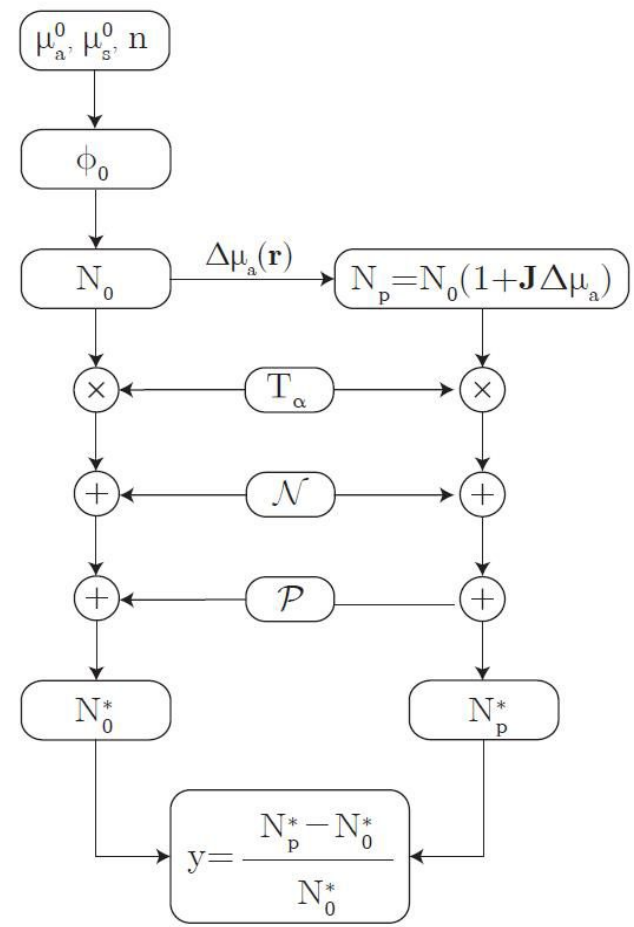

Figure 2. Block diagram of tomographic data generation.

a
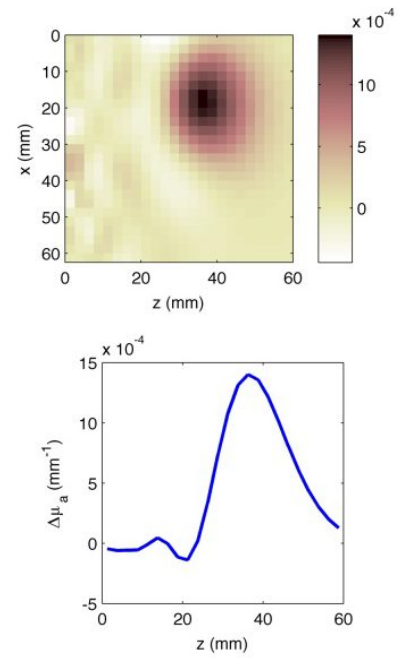
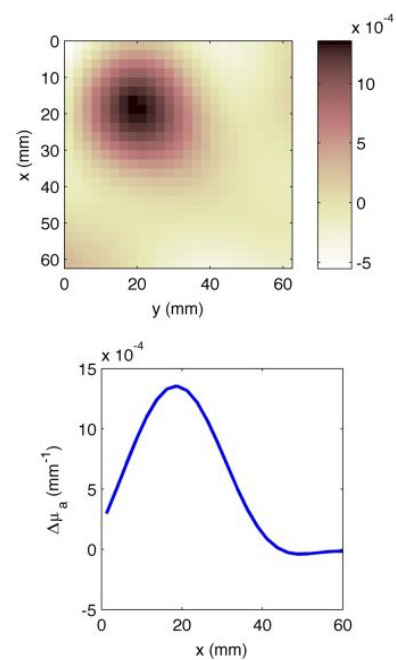

b
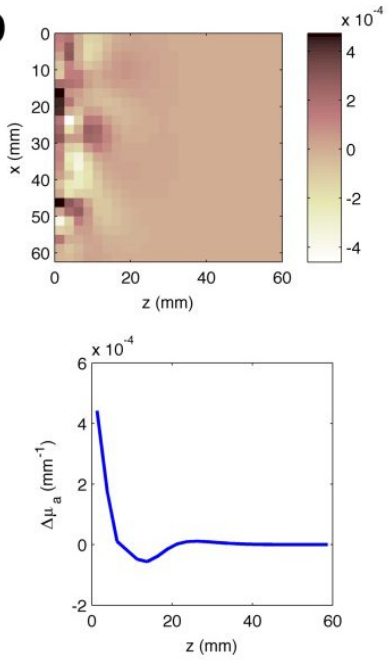
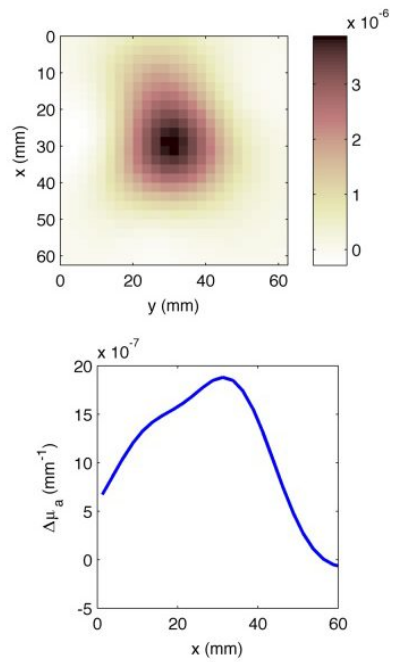

Figure 3. a) TD reconstruction. b) CW reconstruction.

In Figure 3 two slices at the level of the inclusion have been plotted. It is clear that TD is capable to reconstruct the perturbation at the correct position of the perturbation at a depth of $40 \mathrm{~mm}$ whereas $\mathrm{CW}$ reconstructions do not allow a correct localization neither in the $x y$ plane nor in depth.

\section{MINIATURIZED TIME-DOMAIN PROBE}

A dense source-detector arrangement, very close to the ideal case, has already been designed and tested for the CW approach [34]. Instead, in order to fully exploit the tremendous TD potential shown in previous simulations, three requirements remains to be addressed: i) the use of a dense distribution of pulsed sources (condition $\mathrm{C} 1$ ) so as to maximize the injected power (in accordance with the maximum permissible exposure regulation for safety); ii) a similar 
dense arrangement of time-resolved detectors (condition C2) so as to maximize the photon collection; an efficient timegating capability (condition C3) so to extract late photons out of the overwhelming amount of weakly diffused photons at null- $\rho$. In order to fulfill each one of these requirements, we realized two optical probes exploiting recent technology achievements.

Figure 4 (left) shows one of the developed compact single-channel TD probes, for small source-detector separation measurements. The printed circuit board hosts both a pulsed Vertical-Cavity Surface-Emitting Laser [35] (front-end electronics developed at Politecnico di Milano, VCSEL Part number 680C, Vixar Inc., USA) and a fast-gated CMOS Single-Photon Avalanche Diode (SPAD, developed at Politecnico di Milano, Italy [36]), together with the required frontend circuit. The VCSEL provides $<300$ ps optical pulses at MHz rate. External pulse generators are required to trigger the laser emission from VCSEL and to fast-gate the CMOS SPAD. This device demonstrates the possibility to integrate microelectronic pulsed lasers directly onto the probe, thus enabling a dense packing of VCSELs (i.e. to fulfill C1) provided that suitable circuits are designed to replace the external pulse generator. Additionally, the probe-hosted detector demonstrates condition C3 (effective gating capability) on a single SPAD element. Again, suitable electronics must be designed to replace the external gate-signal generator, but CMOS SPADs easily allow the integration of auxiliary electronic circuits close to the single-photon detector. Unfortunately, even using large area SPADs, the collection area of single elements is limited to $0.03 \mathrm{~mm}^{2}$. Hence, in order to demonstrate condition $\mathrm{C} 2$, we designed a different probe based on a Silicon Photomultiplier (SiPM) [37] (model C30742-11 by Excelitas Technologies). This kind of device is essentially an array of hundreds SPADs arranged a dense matrix. SiPM development has been mainly driven by positron emission tomography (PET) systems, Cerenkov telescopes and high-energy physics in general [38], applications in which a large number of devices must be arranged in dense structures, so to cover a wide area by using hundreds or thousands single SiPMs. This requirement is similar to the condition C2 for diffuse optics.

Preliminary characterization on phantom $\left(\mu_{a}=0.1 \mathrm{~cm}^{-1}, \mu_{s}^{\prime}=10 \mathrm{~cm}^{-1}\right)$ have been performed using both probes, demonstrating exciting results. Figure 4 (right) shows the relative contrast $C$ for different $z$ positions of the inclusion (black plastic cylinder having $5 \mathrm{~mm}$ diameter and $5 \mathrm{~mm}$ height, it simulates a $160 \%$ absorption increase over a $1 \mathrm{~cm}^{3}$ sphere [39]), calculated within a time window of $500 \mathrm{ps}$ along the time-resolved reflectance curve placed at a delay so as to maximize contrast for the deepest positions of the inclusion. For the configuration using a VCSEL and a fast-gated SPAD a distance $\rho=5 \mathrm{~mm}$ was selected. Vice versa, for the configuration with VCSEL and SiPM, a $\rho=3 \mathrm{~cm}$ distance was used due to the lack of gating capability. It is worth noting that, even in this first implementation, both systems allow to detect the inclusion well above $2 \mathrm{~cm}$ depth, thus permitting to easily reach, for example, the adult brain cortex. However, further investigations are needed to fully characterize these devices and the possible improvements with respect to state of the art technology.
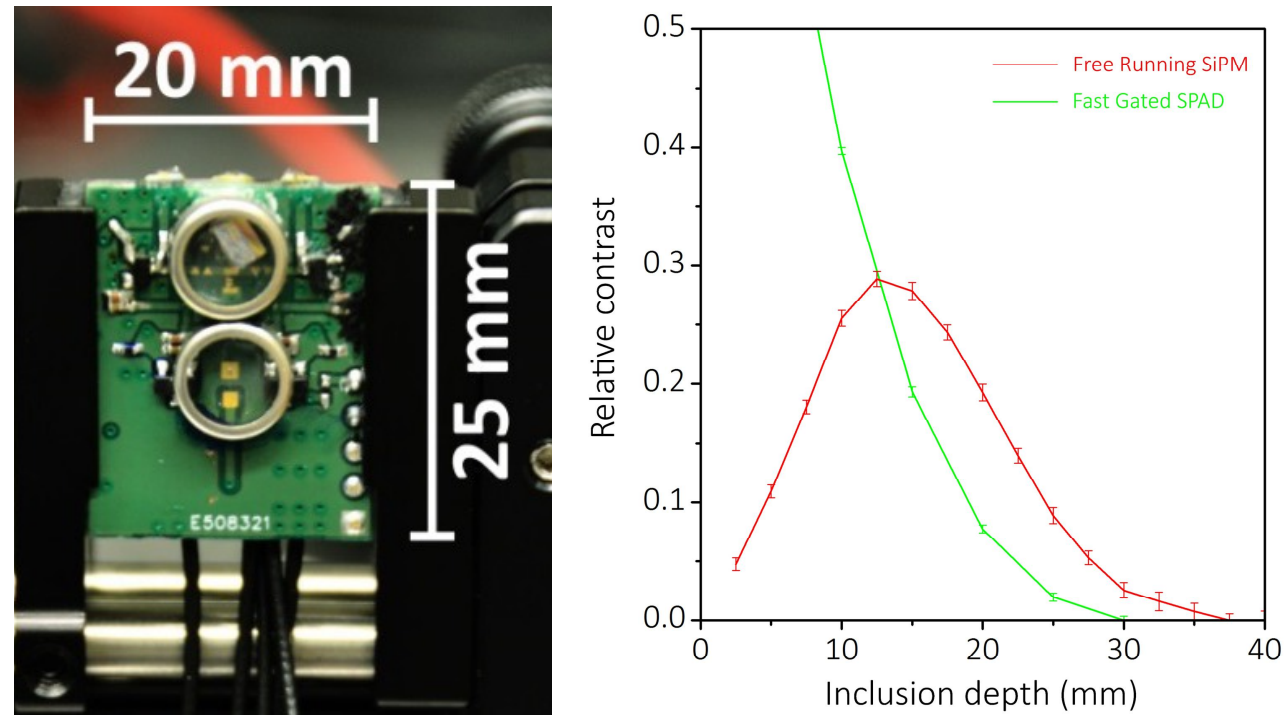

Figure 4. Optoelectronic probe for small source-detector separation: a VCSEL and a $200 \mu \mathrm{m}$ active area diameter CMOSSPAD (placed at $5 \mathrm{~mm}$ distance) are hosted together with the front-end circuitry (left). Relative contrasts obtained for different $z$ positions of a totally absorbing inclusion (right). 


\section{CONCLUSIONS}

In this work we have provided evidence that an ideal time-domain diffuse optics system can obtain a 3-fold increase in the maximum depth, thus possibly reaching $6 \mathrm{~cm}$ in localizing absorbance perturbations. Additionally, we demonstrated that ideal TD systems can overcome ideal CW devices also in tomographic applications. Finally, we identified suitable technologies that potentially allow to address all the requirements needed to push TD diffuse optics close to its physical limits, that are: $i$ ) VCSELs, to arrange many lasers in a dense structure so to maximize the injected power; ii) SiPMs, since they can be easily arranged in a dense matrix to maximize the medium coverage so to increase the photon collection with respect to state of the art devices; iii) fast-gated CMOS-SPADs, thus demonstrating the gating capability onto the SiPM basic element. These new technologies can open the way towards the design of first compact and wearable TD diffuse optical imaging devices for a widespread use in real life.

\section{REFERENCES}

[1] Torricelli, A., Contini, D., Pifferi, A., Caffini, M., Re, R., Zucchelli, L., Spinelli, L., "Time domain functional NIRS imaging for human brain mapping," Neuroimage 85(1), 28-50 (2014).

[2] Torricelli, A., Contini, D., Dalla Mora, A., Pifferi, A., Re, R., Zucchelli, L., Caffini, M., Farina, A., Spinelli, L., "Neurophotonics: noninvasive optical techniques for monitoring brain functions," Functional Neurology 29(4), $1-8$ (2015).

[3] Scholkmann, F., Kleiser, S., Metz, A.J., Zimmermann, R., Mata Pavia, J., Wolf, U., Wolf, M., "A review on continuous wave functional near-infrared spectroscopy and imaging instrumentation and methodology," Neuroimage 85(1), 6-27 (2014).

[4] Wolf, M., Ferrari, M., Quaresima, V., "Progress of near-infrared spectroscopy and topography for brain and muscle clinical applications," J. Biomed. Opt. 12(6), 062104 (2007).

[5] Pifferi, A., Farina, A., Torricelli, A., Quarto, G., Cubeddu, R., Taroni, P., "Review: Time-domain broadband near infrared spectroscopy of the female breast: a focused review from basic principles to future perspectives," J. Near Infrared Spectrosc. 20(1), 223-235 (2012).

[6] Torricelli, A., Spinelli, L., Contini, D., Vanoli, M., Rizzolo, A., Eccher Zerbini, P., "Time-resolved reflectance spectroscopy for non-destructive assessment of food quality," Sens. \& Instrumen. Food Qual. 2, 82-89 (2008).

[7] Bargigia, I., Nevin, A., Farina, A., Pifferi, A., D’Andrea, C., Karlsson, M., Lundin, P., Somesfalean, G., Svanberg, S., "Diffuse optical techniques applied to wood characterisation," J. Near Infrared Spectrosc. 21, 259-268 (2013).

[8] Khoptyar, D., Subash, A.A., Johansson, S., Saleem, M., Sparén, A., Johansson, J., Andersson-Engels, S., "Broadband photon time-of-flight spectroscopy of pharmaceuticals and highly scattering plastics in the VIS and close NIR spectral ranges," Opt. Express 21(17), 20941-20953 (2013).

[9] Maas, A.I.R., Citerio, G., "Noninvasive monitoring of cerebral oxygenation in traumatic brain injury: a mix of doubts and hope," Intensive Care Med. 36, 1283-1285 (2010).

[10] Ghosh, A., Elwell, C., Smith, M., "Cerebral near-infrared spectroscopy in adults: a work in progress," Anesthesia \& Analgesia 115(6), 1373-1383 (2012).

[11] Patterson, M.S., Chance, B., Wilson, B.C., "Time resolved reflectance and transmittance for the non-invasive measurement of tissue optical properties," Appl. Opt. 28(12), 2331-2336 (1989).

[12] Steinbrink, J., Wabnitz, H., Obrig, H., Villringer, A., Rinneberg, H., "Determining changes in NIR absorption using a layered model of the human head," Phys. Med. Biol. 46(3), 879-896 (2001).

[13] Selb, J., Stott, J.J., Franceschini, M.A., Sorensen, A.G., Boas, D.A., "Improved sensitivity to cerebral hemodynamics during brain activation with a time-gated optical system: analytical model and experimental validation," J. Biomed. Opt. 10(1), 011013 (2005).

[14] Torricelli, A., Pifferi, A., Spinelli, L., Cubeddu, R., Martelli, F., Del Bianco, S., Zaccanti, G., "Time-resolved reflectance at null source-detector separation: improving contrast and resolution in diffuse optical imaging," Phys. Rev. Lett. 95(7), 078101 (2005).

[15] Pifferi, A., Torricelli, A., Spinelli, L., Contini, D., Cubeddu, R., Martelli, F., Zaccanti, G., Tosi, A., Dalla Mora, A., Zappa, F., Cova, S., "Time-resolved diffuse reflectance using small source-detector separation and fast singlephoton gating," Phys. Rev. Lett. 100(13), 138101 (2008).

[16] Puszka, A., Di Sieno, L., Dalla Mora, A., Pifferi, A., Contini, D., Boso, G., Tosi, A., Hervé, L., Planat-Chrétien, A., Koenig, A., Dinten, J.M., "Time-resolved diffuse optical tomography using fast-gated single-photon avalanche diodes," Biomed. Opt. Express 4(8), 1351 - 1365 (2013). 
[17] Mazurenka, M., Di Sieno, L., Boso, G., Contini, D., Pifferi, A., Dalla Mora, A., Tosi, A., Wabnitz, H., Macdonald, R., "Non-contact in vivo diffuse optical imaging using a time-gated scanning system," Biomed. Opt. Express 4(10), $2257-2268$ (2013).

[18] Puszka, A., Di Sieno, L., Dalla Mora, A., Pifferi, A., Contini, D., Planat-Chrétien, A., Koenig, A., Boso, G., Tosi, A., Hervé, L., Dinten, J.-M., "Spatial resolution in depth for time-resolved diffuse optical tomography using short source-detector separations," Biomed. Opt. Express 6(1), 1 - 10 (2015).

[19] Contini, D., Dalla Mora, A., Spinelli, L., Farina, A., Torricelli, A., Cubeddu, R., Martelli, F., Zaccanti, G., Tosi, A., Boso, G., Zappa, F., Pifferi, A., "Effects of time-gated detection in diffuse optical imaging at short source-detector separation,” J. Phys. D: Appl. Phys. 48(4), 045401 (2015).

[20] Alerstam, E., Svensson, T., Andersson-Engels, S., Spinelli, L., Contini, D., Dalla Mora, A., Tosi, A., Zappa, F., Pifferi, A., "Single-fiber diffuse optical time-of-flight spectroscopy," Opt.Lett. 37(14), 2877-2879 (2012).

[21] Dalla Mora, A., Contini, D., Pifferi, A., Cubeddu, R., Tosi, A., Zappa, F., "Afterpulse-like noise limits dynamic range in time-gated applications of thin-junction silicon single-photon avalanche diode," Appl. Phys. Lett. 100(24), 241111 (2012).

[22] Boso,G., Dalla Mora, A., Della Frera, A., Tosi, A., "Fast-gating of Single-Photon Avalanche Diodes with 200 ps transitions and 30 ps timing jitter," Sens. Actuators A 191, $61-67$ (2013).

[23] Buttafava, M., Boso, G., Ruggeri, A., Dalla Mora, A., Tosi, A., "Time-gated single-photon detection module with 110 ps transition time and up to $80 \mathrm{MHz}$ repetition rate," Rev. Sci. Instrum. 85(8), 083114 (2014).

[24] Re, R., Contini, D., Turola, M., Spinelli, L., Zucchelli, L., Caffini, M., Cubeddu, R., Torricelli, A., "Multi-channel medical device for time domain functional near infrared spectroscopy based on wavelength space multiplexing," Biomed. Opt. Express 4(10), 2231-2246 (2013).

[25] Carraresi, S., Shatir, T.S.M., Martelli, F., Zaccanti, G., "Accuracy of a perturbation model to predict the effect of scattering and absorbing inhomogeneities on photon migration," Appl. Opt. 40, 4622-4632 (2001).

[26] Contini, D., Martelli, F., Zaccanti, G., "Photon migration through a turbid slab described by a model based on diffusion approximation. I. Theory," Appl. Opt. 36, 4587-4599 (1997).

[27] Vera, M.U., Durian, D.J., “Angular distribution of diffusely transmitted light,” Phys. Rev. E 53, 3215-3224 (1996).

[28] Martelli, F., Sassaroli, A., Zaccanti, G., Yamada, Y., "Properties of the light emerging from a diffusive medium: angular dependence and flux at the external boundary," Phys. Med. Biol. 44, 1257-1275 (1999).

[29] Martelli, F., Del Bianco, S., Ismaelli, A., Zaccanti, G., [Light propagation through biological tissue and other diffusive media], SPIE Press, Washington, USA (2010).

[30] Dalla Mora, A., Tosi, A., Zappa, F., Cova, S., Contini, D., Pifferi, A., Spinelli, L., Torricelli, A., Cubeddu, R., "Fastgated single-photon avalanche diode for wide dynamic range near infrared spectroscopy," IEEE J. Sel. Top. Quantum Electron. 16, 1023-1030 (2010).

[31] Tosi, A., Dalla Mora, A., Zappa, F., Gulinatti, A., Contini, D., Pifferi, A., Spinelli, L., Torricelli, A., Cubeddu, R., "Fast-gated single-photon counting technique widens dynamic range and speeds up acquisition time in time-resolved measurements," Opt. Express 19(11), 10735-10746 (2011).

[32] Press, W.H., Teukolsky, S.A., Vetterling, W.T., Flannery, B.P., [Numerical recipes: the art of scientific computing], Cambridge University Press, Cambridge (1988).

[33] Arridge, S. R., “Optical tomography in medical imaging,” Inverse Probl. 15, R41-R93 (1999).

[34] Eggebrecht, A.T., Ferradal, S.L., Robichaux-Viehoever, A., Hassanpour, M.S., Dehghani, H., Snyder, A.Z., Hershey, T., Culver, J.P. "Mapping distributed brain function and networks with diffuse optical tomography," Nat. Photonics 8, 448-454 (2014).

[35] Johnson, K., Hibbs-Brenner, M., Hogan, W., Dummer, M., “Advances in red VCSEL technology," Adv. Opt. Technol. 2012, 1-13 (2012).

[36] Villa, F., Bronzi, D., Zou, Y., Scarcella, C., Boso, B., Tisa, S., Tosi, A., Zappa, F., Durini,D., Weyers, S., Paschen, U., Brockherde, W., "CMOS SPADs with up to $500 \mu \mathrm{m}$ diameter and $55 \%$ detection efficiency at $420 \mathrm{~nm}$," J. Mod. Opt. 61, 102-115 (2014).

[37] Dolgoshein, B., et al., "Status report on silicon photomultiplier development and its applications," Nucl. Instruments Methods Phys. Res. Sect. A Accel. Spectrometers, Detect. Assoc. Equip. 563, 368-376 (2006).

[38] Buzhan, P., et al., "Silicon photomultiplier and its possible applications," Nucl. Inst. Methods Phys. Res. A 504, 4852 (2003).

[39] Martelli, F., Pifferi, A., Contini, D., Spinelli, L., Torricelli, A., Wabnitz, H., MacDonald, R., Sassaroli, A., Zaccanti, G., "Phantoms for diffuse optical imaging based on totally absorbing objects, part 1: Basic concepts," J. Biomed. Opt. 18(6), 66014 (2013). 